\title{
Quasi-Concavity for Gaussian Multicast Relay Channels
}

\author{
Mohit Thakur and Gerhard Kramer \\ Institute for Communications Engineering, Technical University of Munich
}

\begin{abstract}
Standard upper and lower bounds on the capacity of relay channels are cut-set (CS), decode-forward (DF), and quantize-forward (QF) rates. For real additive white Gaussian noise (AWGN) multicast relay channels with one source node and one relay node, these bounds are shown to be quasi-concave in the receiver signal-to-noise ratios and the squared source-relay correlation coefficient. Furthermore, the CS rates are shown to be quasi-concave in the relay position for a fixed correlation coefficient, and the DF rates are shown to be quasi-concave in the relay position. The latter property characterizes the optimal relay position when using $\mathrm{DF}$.
\end{abstract}

\section{INTRODUCTION}

A multicast relay channel (MRC) is an information network with a source node, a relay node, and two or more destination nodes, and where one message originating at the source should be received reliably at the destinations. We consider additive white Gaussian noise (AWGN) MRCs and show that certain information rate expressions are quasi-concave in the receiver signal-to-noise ratios (SNRs), the squared source-relay correlation coefficient, and the relay position. In particular, we study cut-set (CS), decode-forward (DF), and quantize-forward (QF) rates. Quasi-concavity suggests that efficient algorithms can optimize signaling and the relay position.

Relay positioning has been studied by many authors, with a focus on rate enhancement (e.g., [1], [2]), range extension (e.g., [3], [4]), and outage probability (e.g., [1], [5], [6]). We study the problem of placing a relay to maximize the multicast rate by extending results of [7], [8], [9], [10]. A preliminary version of this paper without proofs appeared in [11]. Our focus is on real alphabet channels.

This paper is organized as follows. Section II presents the MRC model and reviews the CS, DF, and QF rates. Section III develops quasi-concavity results in the squared source-relay correlation coefficient $\rho^{2}$ and the channel SNRs. Section IV introduces a distance dependence for the channel gains and shows that the CS rate is quasi-concave in the relay position when $\rho$ is fixed. We further show that the DF rate is quasiconcave in the relay position. Section $\mathrm{V}$ illustrates quasiconcavity for one-, two-, and three-dimensional networks, and compares the performance of two DF strategies. Section VI concludes the paper. The Appendix reviews useful results on concavity and quasi-concavity, and proves a few new results.

\section{A. Model}

\section{MODEL AND INFORMATION RATES}

An MRC has three types of nodes:

- a source node $s$ that generates a message $W$ and transmits the symbols $X_{s}^{n}=X_{s, 1}, X_{s, 2}, \ldots, X_{s, n}$;

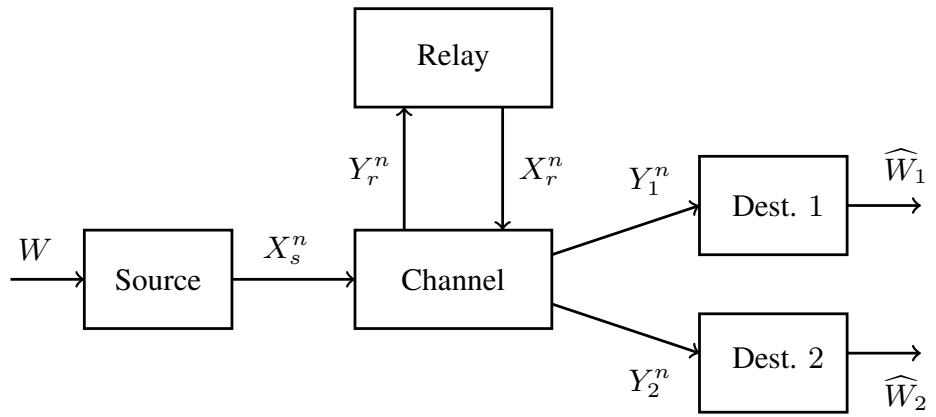

Fig. 1: Multicast relay channel (MRC) with two destinations.

- a relay node $r$ that receives and forwards symbols $Y_{r, k}$ and $X_{r, k}$, respectively, for $k=1,2, \cdots, n$;

- destination nodes $j=1,2, \ldots, N$ where node $j$ receives $Y_{j}^{n}=Y_{j, 1}, Y_{j, 2}, \ldots, Y_{j, n}$ and estimates $W$ as $\widehat{W}_{j}$.

We denote the destination node set as $\mathcal{T}=\{1,2, \ldots, N\}$. The classic relay channel has $N=1$ and Figure 1 shows an MRC with $N=2$.

A memoryless MRC has a function $h(\cdot)$ and a noise random variable $\mathbf{Z}$ so that for every time instant the $N+1$ channel outputs $\mathbf{Y}=\left(Y_{r} Y_{1} \ldots Y_{N}\right)$ are given by

$$
\mathbf{Y}=h\left(X_{s}, X_{r}, \mathbf{Z}\right) \text {. }
$$

The noise $\mathbf{Z}$ is statistically independent of $X_{s}$ and $X_{r}$, and the noise variables at different times are statistically independent.

An encoding strategy for $M$ messages has

- $W$ uniformly distributed over $\{1,2, \ldots, M\}$;

- an encoding function $e_{s}(\cdot)$ such that $X_{s}^{n}=e_{s}(W)$;

- relay functions $e_{r, k}(\cdot)$ with $X_{r, k}=$ $e_{r, k}\left(Y_{r, 1}, \ldots, Y_{r, k-1}\right)$, where $k=1,2, \ldots, n$;

- decoding functions $d_{j}(\cdot)$ such that $d_{j}\left(Y_{j}^{n}\right)=\widehat{W}_{j}, j \in \mathcal{T}$.

The error probability at destination $j$ is $P_{e, j}=$ $\operatorname{Pr}\left[\widehat{W}_{j} \neq W\right]$. The multicast rate is $R=\left(\log _{2} M\right) / n$ bits/use. The rate $R$ is achievable if, for any $\epsilon>0$ and sufficiently large $n$, there is an encoding strategy with $P_{e, j} \leq \epsilon$ for all $j \in \mathcal{T}$. The capacity $C$ is the supremum of the achievable rates.

\section{B. Information Rates}

The following bounds were given in [12] for the relay channel $(N=1)$. Their extensions to MRCs are straightforward.

- CS Rate: $C \leq R_{C S}$ where

$$
R_{C S}=\max \left\{\min _{1 \leq j \leq N} \min \left(I\left(X_{s} X_{r} ; Y_{j}\right), I\left(X_{s} ; Y_{r} Y_{j} \mid X_{r}\right)\right)\right\}
$$




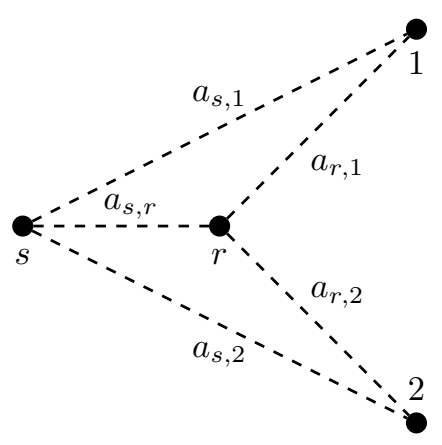

Fig. 2: AWGN MRC with two destinations.

and where the maximization is over all $X_{s} X_{r}$.

- Direct-Transmission (DT) Rate: $C \geq R_{D T}$ where

$$
R_{D T}=\max \left\{\min _{1 \leq j \leq N} I\left(X_{s} ; Y_{j} \mid X_{r}=x^{*}\right)\right\}
$$

and where the maximization is over all $x^{*}$ and $X_{s}$.

- DF Rate: $C \geq R_{D F}$ where

$$
R_{D F}=\max \left\{\min _{1 \leq j \leq N} \min \left(I\left(X_{s} X_{r} ; Y_{j}\right), I\left(X_{s} ; Y_{r} \mid X_{r}\right)\right)\right\}
$$

and where the maximization is over all $X_{s} X_{r}$.

- QF Rate: $C \geq R_{Q F}$ where

$$
\begin{aligned}
& R_{Q F}=\max \left\{\operatorname { m i n } _ { 1 \leq j \leq N } \operatorname { m i n } \left(I\left(X_{s} X_{r} ; Y_{j}\right)-\right.\right. \\
&\left.\left.I\left(Y_{r} ; \widehat{Y}_{r} \mid X_{s} X_{r} Y_{j}\right), I\left(X_{s} ; \widehat{Y}_{r} Y_{j} \mid X_{r}\right)\right)\right\}
\end{aligned}
$$

where $\widehat{Y}_{r}$ is an auxiliary random variable, and where the maximization is over all $X_{s} X_{r} \widehat{Y}_{r}$ such that $X_{s}$ and $X_{r}$ are independent and $X_{s}-X_{r} Y_{r}-\widehat{Y}_{r}$ forms a Markov chain.

\section{Real Alphabet AWGN MRC}

The real alphabet AWGN MRC has real channel symbols and

$$
\begin{aligned}
& Y_{r}=a_{s, r} X_{s}+Z_{r} \\
& Y_{j}=a_{s, j} X_{s}+a_{r, j} X_{r}+Z_{j}
\end{aligned}
$$

where $j \in \mathcal{T}$. The $a_{s, r}, a_{s, j}$, and $a_{r, j}$ are channel gains between the nodes (see Figure 2). We later relate these gains to distances between the nodes. The $Z_{r}$ and $Z_{j}, j=1,2, \ldots, N$, are independent and identically distributed Gaussian random variables with zero mean and unit variance. We may alternatively write (5) and (6) in vector form as

$$
\mathbf{Y}_{j}=\mathbf{A}_{j} \mathbf{X}+\mathbf{Z}_{j}
$$

where $\mathbf{X}=\left(X_{s} X_{r}\right)^{T}, \mathbf{Y}_{j}=\left(Y_{r} Y_{j}\right)^{T}, \mathbf{Z}=\left(Z_{r} Z_{j}\right)^{T}$, and

$$
\mathbf{A}_{j}=\left(\begin{array}{cc}
a_{s, r} & 0 \\
a_{s, j} & a_{r, j}
\end{array}\right) .
$$

We consider individual average block power constraints

$$
\mathrm{E}\left[\sum_{k=1}^{n} X_{s, k}^{2}\right] \leq n P_{s}, \quad \mathrm{E}\left[\sum_{k=1}^{n} X_{r, k}^{2}\right] \leq n P_{r} .
$$

The SNR and the capacity of the link from node $u$ (with transmit power $P_{u}$ ) to node $v$ are the respective

$$
\begin{aligned}
& \mathrm{SNR}_{u, v}=a_{u, v}^{2} P_{u} \\
& \left.\mathrm{C} \mathrm{SNR}_{u, v}\right)=\frac{1}{2} \log \left(1+\mathrm{SNR}_{u, v}\right) .
\end{aligned}
$$

We simplify the above rate bounds for the AWGN MRC.

- CS Rate:

$$
\begin{aligned}
R_{C S}=\max _{\rho} & {\left[\min _{1 \leq j \leq N} \min (\right.} \\
& \left.\mathrm{C}\left(\mathrm{SNR}_{s, j}+\mathrm{SNR}_{r, j}+2 \rho{\left.\sqrt{\mathrm{SNR}_{s, j} \mathrm{SNR}_{r, j}}\right)} \mathrm{C}\left(\left(1-\rho^{2}\right)\left(\mathrm{SNR}_{s, j}+\mathrm{SNR}_{s, r}\right)\right)\right)\right]
\end{aligned}
$$

where the correlation coefficient $\rho$ satisfies $|\rho| \leq 1$. One can restrict attention to non-negative $\rho$.

- DT Rate:

$$
R_{D T}=\min _{1 \leq j \leq N} \mathrm{C}\left(\mathrm{SNR}_{s, j}\right)
$$

- DF Rate:

$$
\begin{aligned}
& R_{D F}=\max _{\rho}\left[\min _{1 \leq j \leq N} \min (\right. \\
& \mathrm{C}\left(\mathrm{SNR}_{s, j}+\mathrm{SNR}_{r, j}+2 \rho \sqrt{\mathrm{SNR}_{s, j} \mathrm{SNR}_{r, j}}\right), \\
&\left.\left.\mathrm{C}\left(\left(1-\rho^{2}\right) \mathrm{SNR}_{s, r}\right)\right)\right] .
\end{aligned}
$$

One can again restrict attention to non-negative $\rho$.

- QF Rate: Optimizing $X_{s} X_{r} \widehat{Y}_{r}$ seems difficult. Instead, we choose $X_{s}$ and $X_{r}$ to be zero-mean Gaussian with variances $P_{s}$ and $P_{r}$, respectively. We further choose $\widehat{Y}_{r}=Y_{r}+Z_{r}$ where $Z_{r}$ is zero-mean Gaussian with variance $N_{r}$. Optimizing $N_{r}$ gives (see [13], pp. 336337)

$$
\widetilde{R}_{Q F}=\min _{1 \leq j \leq N} \mathrm{C}\left(\mathrm{SNR}_{s, j}+\frac{\mathrm{SNR}_{r, j} \mathrm{SNR}_{s, r}}{\mathrm{SNR}_{s, j}+\mathrm{SNR}_{r, j}+\mathrm{SNR}_{s, r}+1}\right) .
$$

\section{A. CS Rate}

\section{QUASI-CONCAVITY IN SNRS AND $\rho^{2}$}

We consider two characterizations of $R_{C S}$. First, let $\mathbf{a}_{j}^{T}=$ $\left(a_{s, j} a_{r, j}\right)$ be the second row of $\mathbf{A}_{j}$, let $\mathbf{Q}_{\mathbf{X}}$ be the covariance matrix of $\mathbf{X}$ (see Appendix $\mathbf{A}$ ), and let $\operatorname{det} \mathbf{M}$ be the determinant of the square matrix $\mathbf{M}$. The CS rate 12 can be expressed as the maximum of

$$
\begin{array}{r}
R_{C S}\left(\mathbf{Q}_{\mathbf{X}}\right)=\min _{1 \leq j \leq N} \min \left(\frac{1}{2} \log \left(\mathbf{a}_{j}^{T} \mathbf{Q}_{\mathbf{X}} \mathbf{a}_{j}+1\right),\right. \\
\left.\frac{1}{2} \log \left(\frac{\operatorname{det} \mathbf{Q}_{\left(\mathbf{Y}_{j}^{T} X_{r}\right)^{T}}}{P_{r}}\right)\right)
\end{array}
$$

over the convex set of $\mathbf{Q}_{\mathbf{X}}$ with diagonal entries $P_{s}$ and $P_{r}$. The first logarithm in $(16)$ is clearly concave in $Q_{\mathbf{X}}$. The second logarithm is concave in $\mathbf{Q}_{\left(\mathbf{Y}_{j}^{T} X_{r}\right)^{T}}$ (see Appendix A and $\mathbf{Q}_{\left(\mathbf{Y}_{j}^{T} X_{r}\right)^{T}}$ is linear in $\mathbf{Q}_{\mathbf{X}}$. To prove the latter claim, observe that

$$
\mathbf{Q}_{\left(\mathbf{Y}_{j}^{T} X_{r}\right)^{T}}=\tilde{\mathbf{A}}_{j} \mathbf{Q}_{\mathbf{X}} \tilde{\mathbf{A}}_{j}^{T}+\left(\begin{array}{cc}
\mathbf{I}_{2} & \mathbf{0} \\
\mathbf{0} & 0
\end{array}\right)
$$


where $\tilde{\mathbf{A}}_{j}^{T}=\left(\mathbf{A}_{j}^{T}\left[\begin{array}{ll}0 & 1\end{array}\right]^{T}\right)$ and $\mathbf{I}_{2}$ is the $2 \times 2$ identity matrix. Hence $R_{C S}\left(\mathbf{Q}_{\mathbf{X}}\right)$ is concave in (the convex set of) $\mathbf{Q}_{\mathbf{X}}$ because it is the minimum of $2 N$ concave functions.

Suppose next that we wish to consider $\rho$ and the SNRs individually rather than via $\mathbf{Q}_{\mathbf{X}}$. Define the vector

$$
\mathbf{S}=\left(\mathrm{SNR}_{s, r}, \mathrm{SNR}_{s, 1}, \cdots, \mathrm{SNR}_{s, N}, \mathrm{SNR}_{r, 1}, \cdots, \mathrm{SNR}_{r, N}\right)
$$

and the functions

$$
\begin{aligned}
& f_{j}(\rho, \mathbf{S})=\mathrm{SNR}_{s, j}+\mathrm{SNR}_{r, j}+2 \rho \sqrt{\mathrm{SNR}_{s, j} \mathrm{SNR}_{r, j}} \\
& g_{j}(\rho, \mathbf{S})=\left(1-\rho^{2}\right)\left(\mathrm{SNR}_{s, j}+\mathrm{SNR}_{s, r}\right) \\
& R_{C S}(\rho, \mathbf{S})=\min _{1 \leq j \leq N} \min \left(\mathrm{C}\left(f_{j}(\rho, \mathbf{S})\right), \mathrm{C}\left(g_{j}(\rho, \mathbf{S})\right)\right) .
\end{aligned}
$$

We establish the following results. We restrict attention to $0 \leq \rho \leq 1$ and positive $\mathbf{S}$.

Lemma 1: $f_{j}(\rho, \mathbf{S})$ and $g_{j}(\rho, \mathbf{S})$ are concave in $\rho$, concave in $\mathbf{S}$, and quasi-concave in $\left(\rho^{2}, \mathbf{S}\right)$.

Proof: Concavity with respect to $\rho$ is established by observing that $f_{j}(\rho, \mathbf{S})$ is linear in $\rho$, and $g_{j}(\rho, \mathbf{S})$ is linear in $-\rho^{2}$ which is concave in $\rho$.

Consider next concavity with respect to $\mathbf{S}$. The Hessian of $f_{j}(\rho, \mathbf{S})$ with respect to $\mathbf{S}$ has only one non-zero eigenvalue

$$
-\frac{\rho}{2} \cdot \frac{\mathrm{SNR}_{s, j}^{2}+\mathrm{SNR}_{r, j}^{2}}{\mathrm{SNR}_{s, j}^{3 / 2} \mathrm{SNR}_{r, j}^{3 / 2}} .
$$

Thus, $f_{j}(\rho, \mathbf{S})$ is concave in $\mathbf{S}$ for non-negative $\rho$ and positive $\mathbf{S}$. The function $g_{j}(\rho, \mathbf{S})$ is linear in $\mathbf{S}$, and thus concave in S.

Now consider quasi-concavity with respect to $\left(\rho^{2}, \mathbf{S}\right)$. Substituting $a=\mathrm{SNR}_{s, j}, b=\mathrm{SNR}_{r, j}, c=\rho^{2}$ into the fifth function of Lemma 5 in Appendix B we find that $f_{j}(\rho, \mathbf{S})$ is quasi-concave in $\left(\rho^{2}, \mathbf{S}\right)$. For the $g_{j}(\rho, \mathbf{S})$, observe that $a b$ is quasi-concave for non-negative $(a, b)$, see the first function of Lemma 5. This implies

$$
\left(\lambda a_{1}+\bar{\lambda} a_{2}\right)\left(\lambda b_{1}+\bar{\lambda} b_{2}\right) \geq \min \left(a_{1} b_{1}, a_{2} b_{2}\right)
$$

for $0 \leq \lambda \leq 1$, and where $\bar{\lambda}=1-\lambda$. Substituting $a_{i}=1-\rho_{i}^{2}$ and $b_{i}=\operatorname{SNR}_{s, j, i}+\operatorname{SNR}_{s, r, i}$ for $i=1,2$, we find that $g_{j}(\rho, \mathbf{S})$ is quasi-concave in $\left(\rho^{2}, \mathbf{S}\right)$.

Theorem 1: $R_{C S}(\rho, \mathbf{S})$ is concave in $\rho$, concave in $\mathbf{S}$, and quasi-concave in $\left(\rho^{2}, \mathbf{S}\right)$.

Proof: $R_{C S}(\rho, \mathbf{S})$ involves taking logarithms and minima of (quasi-) concave functions. The results thus follow by applying Lemma 1 above and Lemma 4, Parts 2 and 3, in Appendix B

Corollary 1: Consider $\mathbf{S}$ as a function of $\mathbf{P}=\left(P_{s}, P_{r}\right)$. Then $R_{C S}(\rho, \mathbf{S}(\mathbf{P}))$ is quasi-concave in $\left(\rho^{2}, \mathbf{P}\right)$.

Proof: The proof follows from the proof of Theorem 1 and because $\mathbf{S}$ is a linear function of $\mathbf{P}$.

\section{B. DF Rate}

Consider the functions

$$
\begin{aligned}
& g_{j}^{*}(\rho, \mathbf{S})=\left(1-\rho^{2}\right) \mathrm{SNR}_{s, r} \\
& R_{D F}(\rho, \mathbf{S})=\min _{1 \leq j \leq N} \min \left(\mathrm{C}\left(f_{j}(\rho, \mathbf{S})\right), \mathrm{C}\left(g_{j}^{*}(\rho, \mathbf{S})\right)\right) .
\end{aligned}
$$

As above, we restrict attention to $0 \leq \rho \leq 1$ and positive $\mathbf{S}$.

Theorem 2: $R_{D F}(\rho, \mathbf{S})$ is concave in $\rho$, concave in $\mathbf{S}$, and quasi-concave in $\left(\rho^{2}, \mathbf{S}\right)$.

Proof: The proof is similar to that of Theorem 1

Corollary 2: $R_{D F}(\rho, \mathbf{S}(\mathbf{P}))$ is quasi-concave in $\left(\rho^{2}, \mathbf{P}\right)$.

Proof: See the proof of Corollary 1 .

\section{DT Rate}

The DT rate (13) is clearly concave in $\mathbf{S}$ and $\mathbf{P}$.

\section{QF Rate}

Consider the functions

$$
\begin{aligned}
& h_{j}(\mathbf{S})=\mathrm{SNR}_{s, j}+\frac{\mathrm{SNR}_{r, j} \mathrm{SNR}_{s, r}}{\mathrm{SNR}_{s, j}+\mathrm{SNR}_{r, j}+\mathrm{SNR}_{s, r}+1} \\
& \widetilde{R}_{Q F}(\mathbf{S})=\min _{1 \leq j \leq N} \mathrm{C}\left(h_{j}(\mathbf{S})\right) .
\end{aligned}
$$

We establish the following results. We restrict attention to nonnegative $\mathbf{S}$.

Lemma 2: $h_{j}(\mathbf{S})$ is quasi-concave in $\left(\mathrm{SNR}_{r, j}, \mathrm{SNR}_{s, r}\right)$.

Proof: Substitute $a=\mathrm{SNR}_{r, j}, b=\mathrm{SNR}_{s, r}, k=\mathrm{SNR}_{s, j}+1$ into the second function of Lemma 5 in Appendix $B$, and apply Lemma 4. Part 1 .

Theorem 3: $R_{Q F}(\mathbf{S})$ is quasi-concave in $\mathbf{S}$ if the $\mathrm{SNR}_{s, j}$, $j=1,2, \ldots, n$, are held fixed.

Proof: Apply Lemma 2 above and Lemma 4 Parts 2 and 3 in Appendix B.

\section{QUASI-CONCAVITY IN RELAY POSITION}

Suppose the channel gain for the node pair $(i, j)$ is

$$
a_{i, j}=\sqrt{\xi_{i, j}} / D_{i, j}^{\alpha / 2}
$$

where $\xi_{i, j}$ is a "fading" gain, $D_{i, j}=\|\mathbf{i}-\mathbf{j}\|$ is the Euclidean distance between the positions $\mathbf{i}$ and $\mathbf{j}$ of nodes $i$ and $j$, respectively, and $\alpha \geq 2$ is a path-loss exponent. We thus have

$$
\mathrm{SNR}_{i, j}=\frac{\xi_{i, j} P_{i}}{D_{i, j}^{\alpha}}=\frac{\xi_{i, j} P_{i}}{\|\mathbf{i}-\mathbf{j}\|^{\alpha}} .
$$

We establish quasi-concavity results in $\rho^{2}$ and $\mathbf{r}$, where $\mathbf{r}$ is the position of the relay node.

\section{A. CS Rate}

Consider the functions (19)-21) but relabeled as $f_{j}(\rho, \mathbf{r})$, $g_{j}(\rho, \mathbf{r})$, and $R_{C S}(\rho, \mathbf{r})$ to emphasize the dependence on the considered parameters. We again consider $0 \leq \rho \leq 1$ and positive $\mathbf{S}$.

Lemma 3: $f_{j}(\rho, \mathbf{r})$ and $g_{j}(\rho, \mathbf{r})$ are quasi-concave in $\mathbf{r}$ for fixed $\rho$. Furthermore, $f_{j}(\rho, \mathbf{r})$ is quasi-concave in $\left(\rho^{2}, \mathbf{r}\right)$.

Proof: Consider the functions

$$
\begin{aligned}
& \tilde{f}_{j}\left(\rho, D^{\alpha}\right)=\frac{\xi_{s, j} P_{s}}{D_{s, j}^{\alpha}}+\frac{\xi_{r, j} P_{r}}{D^{\alpha}}+2 \rho \sqrt{\frac{\xi_{s, j} P_{s}}{D_{s, j}^{\alpha}} \frac{\xi_{r, j} P_{r}}{D^{\alpha}}} \\
& \tilde{g}_{j}\left(\rho, D^{\alpha}\right)=\left(1-\rho^{2}\right)\left(\frac{\xi_{s, j} P_{s}}{D_{s, j}^{\alpha}}+\frac{\xi_{s, r} P_{s}}{D^{\alpha}}\right)
\end{aligned}
$$


which are quasi-linear in $D^{\alpha}$ for fixed $\rho$ since they are decreasing in $D^{\alpha}$. However, $D_{r, j}^{\alpha}$ is a convex function of $\mathbf{r}$ for $\alpha \geq 1$, and thus Lemma 4, Part 5, in Appendix B establishes that $f_{j}(\rho, \mathbf{r})$ is quasi-concave in $\mathbf{r}$ for fixed $\rho$. Similarly, $D_{s, r}^{\alpha}$ is a convex function of $\mathbf{r}$ for $\alpha \geq 1$, and we find that $g_{j}(\rho, \mathbf{r})$ is quasi-concave in $\mathbf{r}$ for fixed $\rho$.

Next, substitute $a=D^{\alpha}$ and $b=\rho^{2}$ into the third function of Lemma 5 , and use Lemma 4 Part 1 , to show that $\tilde{f}_{j}\left(\rho, D^{\alpha}\right)$ is quasi-concave in $\left(\rho^{2}, D^{\alpha}\right)$. However, $\tilde{f}_{j}$ is decreasing in $D^{\alpha}$ and $D_{r, j}^{\alpha}$ is convex in $\mathbf{r}$, so Lemma 4, Part 5, establishes that $f_{j}(\rho, \mathbf{r})$ is quasi-concave in $\left(\rho^{2}, \mathbf{r}\right)$.

Unfortunately, $\tilde{g}_{j}$ is quasi-convex (and not quasi-concave) in $\left(\rho^{2}, D^{\alpha}\right)$. To see this, substitute $a=D^{\alpha}$ and $b=\rho^{2}$ into the fourth function of Lemma 5 . Quasi-concavity would have been useful since it would have permitted using Lemma 4 Parts 2 and 4 , to establish the quasi-concavity of

$$
R_{C S}(\mathbf{r})=\max _{\rho}\left[\min _{1 \leq j \leq N} \min \left(\mathrm{C}\left(f_{j}(\rho, \mathbf{r})\right), \mathrm{C}\left(g_{j}(\rho, \mathbf{r})\right)\right)\right] .
$$

However, we have been unable to prove this, and our numerical results suggest that $R_{C S}(\rho, \mathbf{r})$ is not quasi-concave in $\left(\rho^{2}, \mathbf{r}\right)$. Nevertheless, Lemma 3 suffices to establish an intermediate result which is useful in Section $\mathrm{V}$ when we study $\rho=0$.

Theorem 4: $R_{C S}(\rho, \mathbf{r})$ is quasi-concave in $\mathbf{r}$ for fixed $\rho$, $0 \leq \rho \leq 1$.

Proof: $R_{C S}(\rho, \mathbf{r})$ is the minimum of functions that are quasiconcave in r. Lemma 4, Part 2, thus establishes the theorem.

\section{B. DF Rate}

The quasi-convexity of $\tilde{g}_{j}\left(\rho, D^{\alpha}\right)$ relaxes for the DF rate (25). Consider the negative of the fourth function of Lemma 5 in Appendix B with $k_{1}=0$ :

$$
f(a, b)=(1-b) k_{2} / a .
$$

This function is quasi-linear in $(a, b)$ since both its superlevel and sublevel sets are convex. This result implies the following theorem. We again consider the functions $24-25$ but relabeled as $g_{j}^{*}(\rho, \mathbf{r})$ and $R_{D F}(\rho, \mathbf{r})$. We further define

$$
\begin{aligned}
& \tilde{g}_{j}^{*}\left(\rho, D^{\alpha}\right)=\left(1-\rho^{2}\right) \frac{\xi_{s, r} P_{s}}{D^{\alpha}} \\
& R_{D F}(\mathbf{r})=\max _{\rho}\left[\min _{1 \leq j \leq N} \min \left(\mathrm{C}\left(f_{j}(\rho, \mathbf{r})\right), \mathrm{C}\left(g_{j}^{*}(\rho, \mathbf{r})\right)\right)\right] .
\end{aligned}
$$

As above, we consider $0 \leq \rho \leq 1$ and positive $\mathbf{S}$.

Theorem 5: $R_{D F}(\rho, \mathbf{r})$ is quasi-concave in $\left(\rho^{2}, \mathbf{r}\right)$, and $R_{D F}(\mathbf{r})$ is quasi-concave in $\mathbf{r}$.

Proof: $\tilde{g}_{j}^{*}\left(\rho, D^{\alpha}\right)$ is quasi-linear in $\left(\rho^{2}, D^{\alpha}\right)$ and decreasing in $D^{\alpha}$. Furthermore, $D_{s, r}^{\alpha}$ is convex in $\mathbf{r}$, and thus Lemma 4 . Part 5 in Appendix B establishes that $g_{j}^{*}(\rho, \mathbf{r})$ is quasi-concave in $\left(\rho^{2}, \mathbf{r}\right) . R_{D F}(\rho, \mathbf{r})$ is therefore quasi-concave in $\mathbf{r}$, as it is the minimum of quasi-concave functions (see Lemma 4. Part 2). Furthermore, $R_{D F}(\mathbf{r})$ is concave in $\mathbf{r}$ by Lemma 4. Part 4

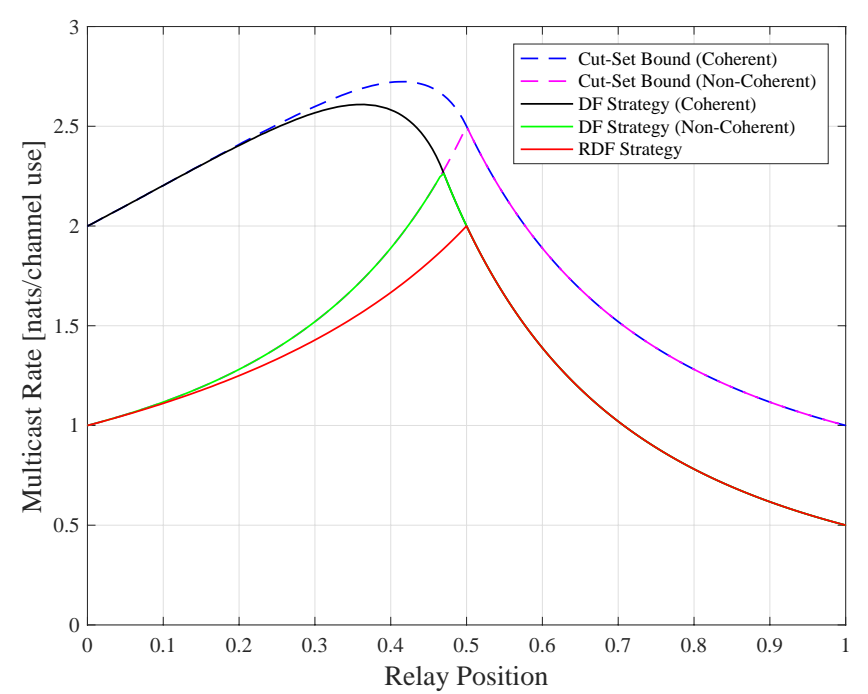

Fig. 3: Relay channel rates for low signal-to-noise ratio (SNR) and $P=1$.

\section{DF PERFORMANCE}

This section presents numerical results for the DF strategy and compares them to results from [7], [8], [9]. We consider 1-, 2-, and 3-dimensional MRCs with different numbers $N$ of destination nodes. For simplicity, we consider the low SNR or broadband regime where

$$
\mathrm{C}(\mathrm{SNR})=\frac{1}{2} \log (1+\mathrm{SNR}) \rightarrow \frac{1}{2} \mathrm{SNR} .
$$

In other words, we consider the CS and DF rates without the logarithms. This approach is valid not only in the limit of low SNR, but more generally because we proved our quasiconcavity results without taking logarithms. Furthermore, in the low SNR regime the rates of full-duplex and half-duplex transmission are the same under a block power constraint.

We choose $P_{s}=P_{r}=P=1, \alpha=2$, and $\xi_{u, v}=1$ for all node pairs $(u, v)$. We study both coherent transmission where $\rho$ is optimized and non-coherent transmission with $\rho=0$. The rates are in nats/channel use. Alternatively, suppose we use sync pulses sampled at $2 W$ samples per second, where $W$ is the (one-sided) signal bandwidth. Suppose further that the (one-sided) noise power spectral density is $1 \mathrm{Wat} / \mathrm{Hz}$. Then at low SNR the rates in nats/channel use are the same as the rates in nats/sec.

\section{A. One Dimension}

Consider a relay channel $(N=1)$ where the source is at the origin $(\mathbf{s}=0)$ and the destination is at point $1(\mathbf{1}=1)$. Figure 3 shows the low SNR CS rates, DF rates, and the routing-based DF (RDF) rates developed in [7]. Observe that all curves are quasi-concave (but not concave) in r. Theorems 4 and 5 predict the quasi-concavity for all curves except for the coherent CS rates. Observe also that the curves for the coherent and non-coherent rates merge for relay positions exceeding a certain value $(r=0.5$ and $r \approx 0.47$ for the respective CS and DF rates). The reason for this behavior is that $\rho=0$ is optimal for the coherent CS and DF rates beyond 
these positions, see the $\rho$ curve in [1] (Figure 16). Furthermore, the non-coherent $\mathrm{CS}$ rates coincide with the non-coherent DF rates for a large range of $\mathbf{r}$.

The best relay positions for the two strategies are different. For example, $\mathbf{r}=0.5$ maximizes $R_{R D F}$ while the $\mathbf{r}$ maximizing $R_{D F}$ is closer to the source. This is because when the source transmits, the relay and the destination listen, and the destination "collects" information. The relay can thus be positioned closer to the source while maintaining the same information rate from the source to the relay, and from the source-relay pair to the destination. At the optimal positions, we compute $R_{D F} \approx 2.26 P$ nats/sec and $R_{R D F}=2 P$ nats/sec, so the DF gain is $\approx 13 \%$.

\section{B. Two Dimensions}

Consider $N=5$ destinations positioned on a square in the two-dimensional Euclidean plane with the source node at the origin. Figure 4 a plots the node positions as circles, and the non-coherent $R_{D F}$ as a function of the relay position. The best relay position is shown by a circle labeled $\mathbf{r}_{\mathrm{DF}}^{*}$ and the corresponding rate is $R_{D F} \approx 0.011 P$ nats/sec. Figure 4 plots the low SNR two-hop rate

$$
R_{2 H} \rightarrow \min _{1 \leq j \leq 5} \frac{1}{2} \min \left(\frac{\xi_{s, r} P_{s}}{\|\mathbf{s}-\mathbf{r}\|^{\alpha}}, \frac{\xi_{r, j} P_{r}}{\|\mathbf{r}-\mathbf{j}\|^{\alpha}}\right)
$$

as a function of the relay position. The best relay position is shown by a circle labeled $\mathbf{r}_{2 H}^{*}$ and the corresponding two-hop rate is $R_{2 H}=0.01 P$ nats/sec. The non-coherent $\mathrm{DF}$ gain is thus $\approx 10 \%$.

Figure 4 p,d shows contour plots for $R_{D F}$ and $R_{2 H}$. The contours form convex regions, as predicted by Theorem 5 . Again, the relay position maximizing $R_{D F}$ lies closer to the source than the relay position maximizing $R_{2 H}$.

\section{CONCLUSIONS}

Various quasi-concavity results were established for AWGN MRCs. In particular, the CS rates are quasi-concave in the relay position for a fixed correlation coefficient (Theorem 4) and the DF rates are quasi-concave in the relay position (Theorem 5).

\section{ACKNOWLEDGMENT}

M. Thakur and G. Kramer were supported by the German Ministry of Education and Research in the framework of an Alexander von Humboldt Professorship.

\section{APPENDIX}

\section{A. Covariance Matrices and Concavity}

The covariance matrix of a real-valued random column vector $\mathbf{V}$ is

$$
\mathbf{Q}_{\mathbf{V}}=\mathrm{E}\left[(\mathbf{V}-\mathrm{E}[\mathbf{V}])(\mathbf{V}-\mathrm{E}[\mathbf{V}])^{T}\right] .
$$

A useful property of covariance matrices is as follows (see [14], p. 684). If $\mathbf{Q}_{\mathbf{V}}^{*}$ is a principal minor of $\mathbf{Q}_{\mathbf{V}}$, then the following function is concave in $Q_{\mathbf{V}}$ :

$$
f\left(\mathbf{Q}_{\mathbf{V}}\right)=\log \frac{\operatorname{det} \mathbf{Q}_{\mathbf{V}}}{\operatorname{det} \mathbf{Q}_{\mathbf{V}}^{*}}
$$

\section{B. Concave and Quasi-Concave Functions}

1) Compositions Preserving Quasi-Concavity: The following compositions preserve quasi-concavity.

Lemma 4: Suppose $f$ and $f_{i}, 1 \leq i \leq n$, are quasi-concave, then so are the functions

1) $h=k_{1} f+k_{2}$, where $k_{1} \geq 0$ and $k_{2} \in \mathbf{R}$;

2) $h=\min _{1<i \leq n} f_{i}$;

3) $h=g \circ f$ where $f$ is quasi-concave and $g$ is nondecreasing;

4) $h(\mathbf{a})=\sup _{\mathbf{b} \in \mathcal{B}} f(\mathbf{a}, \mathbf{b})$ where $\mathcal{B}$ is a convex set;

5) $h(\mathbf{a}, \mathbf{b})=f(g(\mathbf{a}), \mathbf{b})$ where $g$ is convex and $f(\tilde{\mathbf{a}}, \mathbf{b})$ is non-increasing in $\mathbf{a}$ for fixed $\mathbf{b}$.

Proof: Properties 1)-4) are standard (see [15], Section 3.4). For property 5), observe that

$$
\begin{aligned}
& h\left(\lambda \mathbf{a}_{1}+\bar{\lambda} \mathbf{a}_{2}, \lambda \mathbf{b}_{1}+\bar{\lambda} \mathbf{b}_{2}\right) \\
& =f\left(g\left(\lambda \mathbf{a}_{1}+\bar{\lambda} \mathbf{a}_{2}\right), \lambda \mathbf{b}_{1}+\bar{\lambda} \mathbf{b}_{2}\right) \\
& \stackrel{(a)}{\geq} f\left(\lambda g\left(\mathbf{a}_{1}\right)+\bar{\lambda} g\left(\mathbf{a}_{2}\right), \lambda \mathbf{b}_{1}+\bar{\lambda} \mathbf{b}_{2}\right) \\
& \stackrel{(b)}{\geq} \min \left(f\left(g\left(\mathbf{a}_{1}\right), \mathbf{b}_{1}\right), f\left(g\left(\mathbf{a}_{2}\right), \mathbf{b}_{2}\right)\right)
\end{aligned}
$$

where $(a)$ follows because $g\left(\lambda \mathbf{a}_{1}+\bar{\lambda} \mathbf{a}_{2}\right) \leq \lambda g\left(\mathbf{a}_{1}\right)+\bar{\lambda} g\left(\mathbf{a}_{2}\right)$ and $f(\tilde{\mathbf{a}}, \mathbf{b})$ is non-increasing in $\tilde{\mathbf{a}}$. Step $(b)$ follows because $f$ is quasi-concave.

2) Examples of Quasi-Concave Functions: We establish quasi-concavity for several useful functions.

Lemma 5: The following functions are quasi-concave for $\mathbf{x}=(a b)$ with non-negative entries.

1) $f(\mathbf{x})=a b$

2) $f(\mathbf{x})=\frac{a b}{a+b+k}$ for a positive constant $k$

3) $f(\mathbf{x})=k_{1} / a+2 \sqrt{k_{2} b / a}$ for positive constants $k_{1}, k_{2}$

4) $f(\mathbf{x})=-(1-b)\left(k_{1}+k_{2} / a\right)$ for positive constants $k_{1}, k_{2}$, and $b \leq 1$

Furthermore, the following function is quasi-concave for $\mathbf{x}=$ $(a b c)$ with non-negative entries.

5) $f(\mathbf{x})=a+b+2 \sqrt{a b c}$

Proof: We consider positive $\mathbf{x}$, and we use bordered Hessians $\mathbf{B}_{f}(\mathbf{x})$ and the derivatives $D_{k}$ of their $k$ th leading principal minors, $k=2,3, \ldots, n$. The results extend to non-negative $\mathbf{x}$ by using continuity at zero values, except for the third and fourth functions where $a=0$ makes the functions undefined.

1) We have $D_{2}<0$ and $D_{3}>0$ for

$$
\mathbf{B}_{f}(\mathbf{x})=\left(\begin{array}{lll}
0 & b & a \\
b & 0 & 1 \\
a & 1 & 0
\end{array}\right)
$$

2) We have $D_{2}<0$ and $D_{3}>0$ for

$$
\mathbf{B}_{f}(\mathbf{x})=\left(\begin{array}{ccc}
0 & \frac{b(b+k)}{(a+b+k)^{2}} & \frac{a(a+k)}{(a+b+k)^{2}} \\
\frac{b(b+k)}{(a+b+k)^{2}} & \frac{-2 b(b+k)}{(a+b+k)^{3}} & \frac{2 a b+(a+b+k) k}{(a+b+k)^{3}} \\
\frac{a(a+k)}{(a+b+k)^{2}} & \frac{2 a b+(a+b+k) k}{(a+b+k)^{3}} & \frac{-2 a(a+k)}{(a+b+k)^{3}}
\end{array}\right) .
$$

3) We have $D_{2}<0$ and $D_{3}>0$ for

$$
\mathbf{B}_{f}(\mathbf{x})=\left(\begin{array}{ccc}
0 & -\frac{k_{1}+\sqrt{k_{2} a b}}{a^{2}} & \sqrt{\frac{k_{2}}{a b}} \\
-\frac{k_{1}+\sqrt{k_{2} a b}}{a^{2}} & \frac{4 k_{1}+3 \sqrt{k_{2} a b}}{2 a^{3}} & -\frac{\sqrt{k_{2}}}{2 a^{3 / 2} \sqrt{b}} \\
\sqrt{\frac{k_{2}}{a b}} & -\frac{\sqrt{k_{2}}}{2 a^{3 / 2} \sqrt{b}} & -\frac{\sqrt{k_{2}}}{2 b^{3 / 2} \sqrt{a}}
\end{array}\right) .
$$




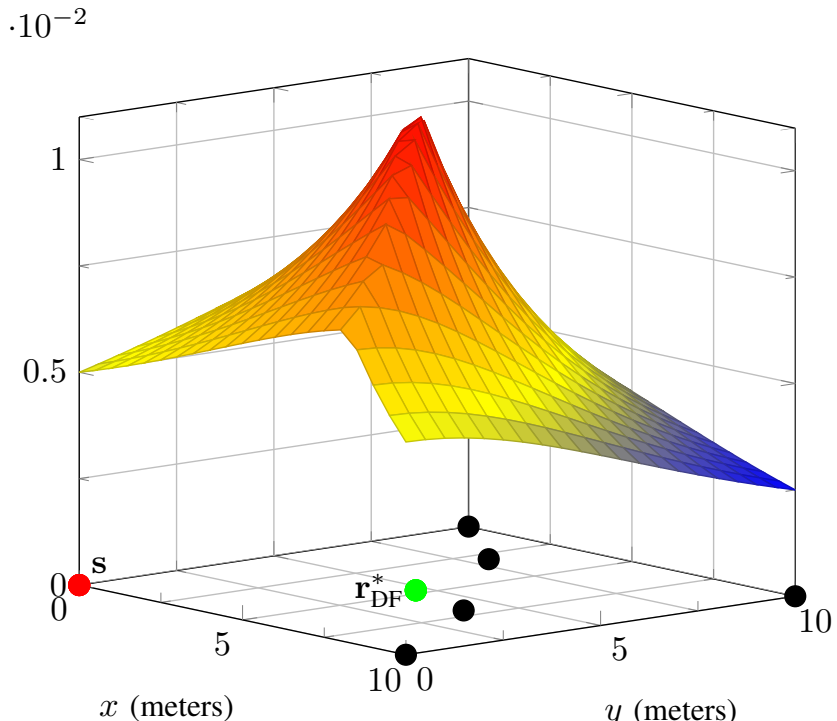

(a)

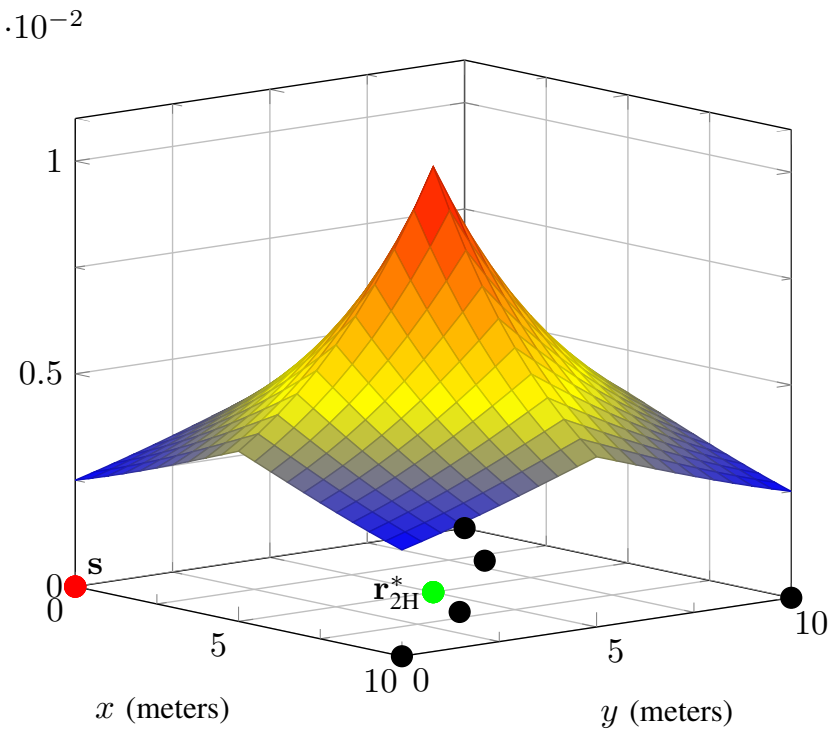

(c)

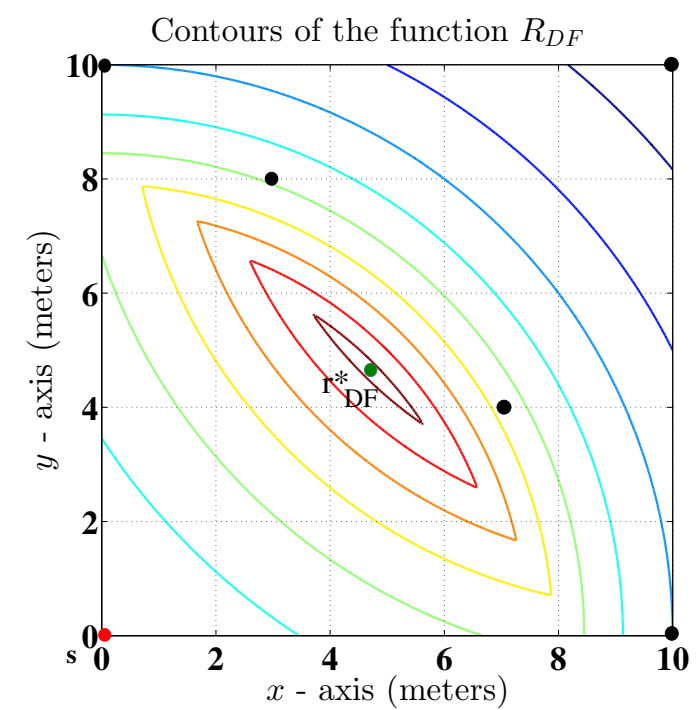

(b)

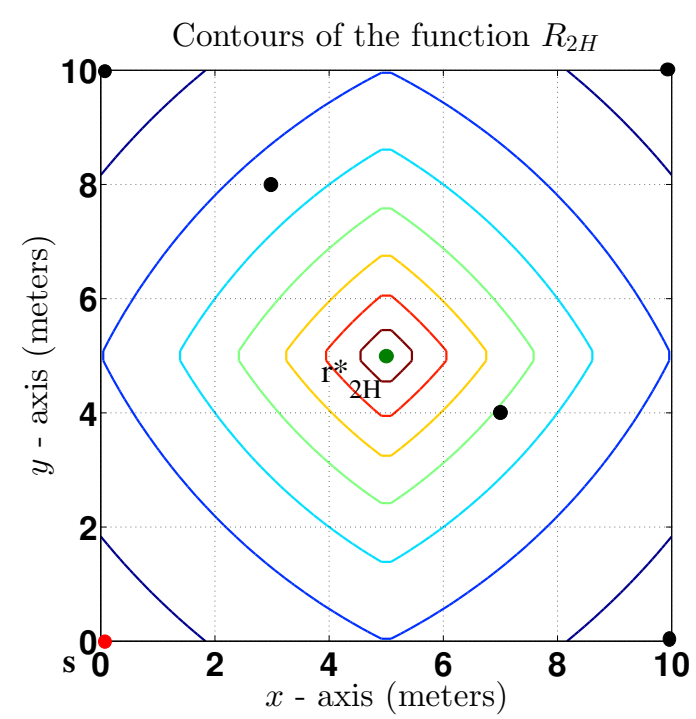

(d)

Fig. 4: (a) $R_{D F}$ for $N=5$; (b) $R_{D F}$ contour plot; (c) $R_{2 H}$ for the same network; (d) $R_{2 H}$ contour plot.

4) If $b \leq 1$, we have $D_{2}<0$ and $D_{3}>0$ for

$$
\mathbf{B}_{f}(\mathbf{x})=\left(\begin{array}{ccc}
0 & \frac{(1-b) k_{2}}{a^{2}} & k_{1}+\frac{k_{2}}{a} \\
\frac{(1-b) k_{2}}{a^{2}} & -\frac{2(1-b) k_{2}}{a^{3}} & -\frac{k_{2}}{a^{2}} \\
k_{1}+\frac{k_{2}}{a} & -\frac{k_{2}}{a^{2}} & 0
\end{array}\right) .
$$

5) We have $D_{2}<0, D_{3}>0$ and $D_{4}<0$ for

$$
\mathbf{B}_{f}(\mathbf{x})=\left(\begin{array}{cccc}
0 & 1+\sqrt{\frac{b c}{a}} & 1+\sqrt{\frac{a c}{b}} & \sqrt{\frac{a b}{c}} \\
1+\sqrt{\frac{b c}{a}} & -\frac{\sqrt{b c}}{2 a^{3 / 2}} & \frac{1}{2} \sqrt{\frac{c}{a b}} & \frac{1}{2} \sqrt{\frac{b}{a c}} \\
1+\sqrt{\frac{a c}{b}} & \frac{1}{2} \sqrt{\frac{c}{a b}} & -\frac{\sqrt{a c}}{2 b^{3 / 2}} & \frac{1}{2} \sqrt{\frac{a}{b c}} \\
\sqrt{\frac{a b}{c}} & \frac{1}{2} \sqrt{\frac{b}{a c}} & \frac{1}{2} \sqrt{\frac{a}{b c}} & -\frac{\sqrt{a b}}{2 c^{3 / 2}}
\end{array}\right) .
$$

\section{REFERENCES}

[1] Kramer, G.; Gastpar, M.; Gupta, P. Cooperative strategies and capacity theorems for relay networks. IEEE Trans. Inf. Theory 2005, 51, 30373063. [CrossRef

[2] Lin, B.; Ho, P.-H.; Xie, L.-L.; Shen, X.; Tapolcai, J. Optimal relay station placement in broadband wireless access networks. IEEE Trans. Mob. Comput. 2010, 9, 259-269. [CrossRef]

[3] Aggarwal, V.; Bennatan, A.; Calderbank, A. R. Calderbank. On maximizing coverage in Gaussian relay channels. IEEE Trans. Inf. Theory 2009, 55, 2518-2536. [CrossRef

[4] Joshi, G.; Karandikar, A. Optimal relay placement for cellular coverage extension. In Nat. Conf. Commun., Bangalore, India, 28-30 January 2011; pp. 11961200.

[5] Lee, J.; Wang, H.; Andrews, J.G.; Hong, D. Outage probability of cognitive relay networks with interference constraints. IEEE Trans. Wirel. Commun. 2011, 10, 390-395. |CrossRef

[6] Chen, X.; Song, S.H.; Letaief, K.B. Relay position optimization improves finite-SNR diversity gain of decode-and-forward MIMO relay systems. IEEE Trans. Commun. 2012, 60, 3311-3321. [CrossRef 
[7] Thakur, M.; Fawaz, N.; Médard, M. Optimal relay location and power allocation for low-SNR broadcast relay channels. IEEE Int.1 Conf. Computer Commun., Shanghai, China, 10-15 April 2011; pp. 28222830.

[8] Thakur, M.; Fawaz, N.; Médard, M. On the geometry of wireless network multicast in 2-D. IEEE Int. Symp. Inf. Theory, Saint Petersburg, Russian, 31 July-5 August 2011; pp. 1628-1632.

[9] Thakur, M.; Fawaz, N.; Médard, M. Reducibility of joint relay positioning and flow optimization problem. IEEE Int. Symp. Inf. Theory, Boston, MA, USA, 1-6 July 2012; pp. 1117-1121.

[10] Thakur, M.; Kramer, G. Relay Positioning for Multicast Relay Networks. IEEE Int. Symp. Inf. Theory, Istanbul, Turkey, 7-12 July 2013; pp. 1954-1958.

[11] Thakur, M.; Kramer, G. Quasi-concavity for Gaussian multicast relay channels. IEEE Int. Symp. Inf. Theory, Hong Kong, China, 14-19 June 2015; pp. 2867-2869.

[12] Cover, T.M.; El Gamal, A. Capacity theorems for the relay channel. IEEE Trans. Inf. Theory 1979, 25, 572-584. |CrossRef|

[13] Kramer, G.; Maric, I.; Yates, R.D. Cooperative Communications. Found. Trends Netw. 2006, 1, 271-425. [CrossRef

[14] Cover, T.M.; Thomas, J.A. Elements of Information Theory, 2nd ed.; John Wiley \& Sons: New York, NY, USA, 2006

[15] Boyd, S.; Vandenberghe, L. Convex Optimization; Cambridge University Press: New York, NY, USA, 2004.

[16] Bazaraa, M.S.; Sherali, H.D.; Shetty, C.M. Nonlinear Programming; Wiley: Hoboken, NJ, USA, 2006 